\title{
MICROTENSILE BOND STRENGTH OF TTEMA/TEGDMA REMINERALIZING ADHE-SIVE TO EARLY CARIOUS ENAMEL LESIONS
}

\author{
Mona M. Ghoneim * and Moustafa N. Aboushelib**
}

\begin{abstract}
Objectives: Resin adhesive could be used as a carrier of nano-hyrdoxy apatite particles added to treat early enamel caries. The aim of this study was to evaluate microtensile bond strength of a remineralizing enamel infiltrant to early carious enamel lesion.

Methods: An artificial caries model was used to induce early partially-cavitated enamel carieslike lesion in maxillay centrals. Nano hydroxyapatite particles (nHA) were applied in two forms to a low shrinkage low viscosity resin adhesive: particles were directly added to resin adhesive or silanated before addition. Unmodified adhesive was used as control. Micro-tensile bond strength (MTBS) was performed to evaluate bond strength to demineralized enamel after application of the remineralizing resin adhesive $(n=20, \alpha=0.05)$. Scanning electron microscopy (SEM) was performed to assess mode of failure and to study infiltrant-enamel interface.
\end{abstract}

Results: Direct addition of nano-particles resulted in significant reduction $(\mathrm{F}=32, \mathrm{P}<0.001)$ in MTBS (19 MPa \pm 3.8 ) compared to addition of silanated particles (24 MPa \pm 3.2 ) which presented comparable values to the unmodified enamel infiltrant (26 MPa \pm 4.1$)$. SEM image analysis revealed adhesive failure observed for directly added particles while the other two groups demonstrated cohesive failure observed in the resin adhesive. On the other hand, direct addition of nHA resulted in partial remineralization of enamel ( $42 \%$ recovery in calcium content).

Conclusions: TTEMA/TEGDMA enamel infiltrant could be used as a carrier for nano hydroxyapatite particles without compromising bond strength to early carious enamel lesions.

KEYWORDS: remineralizing agent, MTBS, SEM, bond, caries, interface, early carious lesion

\footnotetext{
* Associate Professor of Restorative Dentistry, Faculty of Dentistry, Alexandria University, Egypt.

** Professor of Biomaterials, Faculty of Dentistry, Alexandria University, Egypt.
} 


\section{INTRODUCTION}

Remineralization of early carious enamel (ECE) and dentine focused on using different forms of calcium ions as a source of ion exchange across the carious surfaces ${ }^{1}$. Casein phosphopeptide-amorphous calcium phosphate (CPP-ACP) is one of the most successful forms which gained lots of attention in remineralization efforts especially in white spot lesions of enamel. Calcium in an amorphous form is easily transported across porous carious tooth structure and can readily participate in re-crystallization of ion deficient hypomineralized enamel ${ }^{2}$. Different forms of Casein phosphopeptide-amorphous calcium phosphate are currently available in the market. However, application of CPP-ACP has a negative effect on bond strength to treated enam$\mathrm{el}^{3}$. Another approach in treating ECE is sealing the porous enamel lesion using low viscosity resins known as enamel infiltrants ${ }^{4,5}$. Infiltration of non cavitated enamel lesion using different forms of infiltrants have significantly reduced white spot appearance and protected enamel against further demineralization ${ }^{6,7}$. Increasing penetration depth of enamel infiltrants indicated higher efficiency in conservatively treating these lesions ${ }^{8}$. Several attempts focused on loading enamel infiltrants with different forms of remineralizing agents and studied their penetration depth. Addition of fillers of different sizes and concentrations had little effect on the penetration depth of modified enamel infiltrants ${ }^{9}$. Synthetic nano hydroxyapatite (nHA) is considered one of the most biocompatible and bioactive materials, and has gained wide acceptance in medicine and dentistry in recent years. It mimics hydroxyapatite crystals in morphology and structure and arrested mineral loss in vitro. Recently several reports have shown that synthetic nano-hydroxyapatite has some potential in repair and in remineralization of early carious lesions in both enamel and dentin ${ }^{10}$. When added to a tooth paste, synthetic nano hydroxyapatite increased enamel hardness indicating successful ion exchange ${ }^{11}$. Several trials focused on addition of nHA to enamel infiltrants in order to improve its anti-cariogenic properties ${ }^{12,13}$.

If white spots are part of a larger lesion, restoration of cavitated teeth would require a liable bond strength to resin composite. Thus the effect of enamel infiltrant on bond strength was a point worth investigating ${ }^{14}$.

The effect to demineralization/remineralization of bond strength to enamel was previously evaluated $^{15,16}$. Infiltration of demineralized enamel prior to establishing enamel bond improved bond strength and durability ${ }^{17,18}$. However, in order to improve penetration of the infiltrant, surface pretreatments as application of hydrochloric or phosphoric acids are used causing substantial further loss of an already weakened enamel ${ }^{19,20}$.

Using a low viscosity resin as Tris[4-(2'-hydroxy-3'-methacryloyloxypropoxy)phenyl]methane diluted with triethyleneglycol dimethacrylate (TTEMA/TEGDMA) could enhance penetration of ECE without the need to rely on a separate etching procedure $^{21}$. Loading this infiltrant with a remineralizing medium could enhance ion exchange across resin-enamel interface. The aim of this study was to evaluate remineralization potential of a low viscosity enamel infiltrant enriched with nano hydroxy apatite particles and its effect on microtensile bond strength to resin composite.

\section{MATERIALS AND METHODS}

Extracted teeth were collected and demineralized in-vitro to produce early enamel caries-like lesions. Produced lesions were characterized by scanning electron microscopy and cross-sectional hardness testing; Nano-Hydroxyapatite particles were partially hydrolyzed and added to a low viscosity resin before and after silanation. Subsequently composite blocks were bonded to the enamel surface and microtensile bond strength was evaluated. 


\section{Production of caries-like lesions:}

Extracted caries free human maxillary central incisors were collected from the oral \& maxillofacial department, faculty of dentistry, Alexandria university. Teeth were extracted due to periodontal disease from diabetic patients. Teeth were sectioned at cemento-enamel junction level using diamond disc mounted on a precision cutter (Micracut 1.5, Metkon, Turkey). The cut crowns were covered with 2 layers of acid resistant varnish leaving only $4 \times 4 \mathrm{~mm}$ facial window. Demineralizing solution was prepared according to Ten Cate ${ }^{22}$. Briefly the solution was composed of $50 \mathrm{mM}$ acetic acid +2.2 $\mathrm{mM} \mathrm{Ca}(\mathrm{NO} 3) 2.2 \mathrm{H} 2 \mathrm{O}+2.2 \mathrm{mM} \mathrm{KH} 2 \mathrm{PO} 4+0.1$ ppm NaF. The $\mathrm{pH}$ of the solution was adjusted to 4.2 using small amounts of $\mathrm{NaOH}$. The solution $\mathrm{pH}$ was measured daily by $\mathrm{pH}$ meter (Jenway $3020 \mathrm{pH}$ meter, UK) and was kept between 4.2 and 4.25 by adding refreshening amounts of HCL $30 \%$ solution. Teeth were immersed in the demineralizing solution until the center part of the window demonstrated a visibly porous enamel lesion.

\section{Cross-sectional micro hardness test:}

40 Specimens were randomly selected and were embedded in acrylic resin to protect the specimens from any surface damage. The specimens were longitudinally sectioned using a diamond disc mounted in a precision cutter (Micracut 1.5, Metkon, Turkey) under water cooling. Each section was gradually polished using silicon carbide paper ascending grits, $800,1000,1200$, and 2000 to remove any surface damage during sectioning then each block was ultrasonically cleaned in $90 \%$ ethanol. Cross-section micro-hardness was performed using a Vickers indentation point (Wilbert Wilson, Germany) using $200 \mathrm{~g}$ weight applied for 30 seconds. Measurements were taken at from the surface of enamel to a maximum depth of 500 um at 25 um steps. Hardness values of sound enamel was measured and used as a control. This test was repeated after 3 months of water storage to measure the remineralization po- tential of each group tested.

\section{Assessment of morphological changes and cal- cium loss:}

Carious enamel specimens were gold-sputter coated (Fine coat jfc-1100E, Jeol, Munich, Germany) and examined using scanning electron microscope (JSM-5300, Jeol, Munich, Germany). Calcium and phosphorus contents were evaluated from the surface of carious enamel to a maximum depth of $300 \mathrm{um}$ at $25 \mathrm{um}$ steps using Energy-dispersive $\mathrm{X}$-ray spectroscopy (EDX) unit and average spot size of 1 um.

\section{Preparation of nano-hydroxyapatite infiltrant (nHA)}

Nano hydroxyapatite particles were prepared by sol gel chemical reaction. Particle size and distribution were evaluated using particle size analyzer and particles size in range of 40-60 nanometers was selected. The surface of the artificially created white spot was etched with a mixture of $2 \%$ chlorhexidine and $10 \%$ sodium hypochlorite for $2 \mathrm{~min}$. The teeth were finally washed and dried. Low viscosity TTEMA/TEGDMA resin infiltration was prepared by first preparing Tris[4-(2'-hydroxy-3'-methacryloyloxypropoxy)phenyl]methane (TTEMA) by mixing triphenylolmethane triglycidyl ether (TTE) with methacrylic acid (MA) in the presence of 4-(dimethylamino)pyridine. 50\% TEGDMA was added to the mixture with $0.1 \%$ photo initiator ${ }^{23}$.

The particles were partially hydrolyzed using $10 \%$ phosphoric acid and $10 \%$ wt nHA powder was either directly mixed with the prepared infiltrant or coated with a silane coupling agent (Silane coupling agent, 3M ESPE, St Paul, MN, USA) before addition to the resin infiltrant. A coat of was applied using a microbrush and light polymerized using $2500 \mathrm{~mW}$ LED device. A layer $3 \mathrm{~mm}$ thick of resin composite (Filteck z350, 3M ESPE, USA) was added and polymerized on the demineralized facial surface. To evaluate calcium uptake, some 
specimens were stored under demineralized water before calcium content was evaluated as previously discussed.

\section{Microtensile bond strength test (MTBS)}

Microbars were prepared by cutting bonded specimens using precision cutting machine (Isomet 1000, Buehler, Lake Bluff, Ill, USA). 25 microbars, $1 \times 1 \times 5 \mathrm{~mm}$, were chosen randomly from cut specimens. All specimens were loaded to failure using universal testing machine (Instron 6022, Instron Limited, High Wycombe, UK) at constant crosshead speed of $0.5 \mathrm{~mm} / \mathrm{min}$. Fractured microbars were examined under stereo-microscope (SZ 11, Olympus Inc, Osaka, Japan) to detect failure mode ${ }^{24}$.

\section{Scanning electron microscopy (SEM):}

Intact sections were collected during sectioning procedure as described previously. The sections were polished using silicon carbide paper (800, 1000 , and 1200 grit). The sections were ultrasonically cleaned in demineralized water for $15 \mathrm{~min}$ and dried at $600 \mathrm{C}$ for $60 \mathrm{~min}$. Sections were gold sputter coated and prepared for SEM examination (XL 30, Phillips, Eindhoven, the Netherlands).

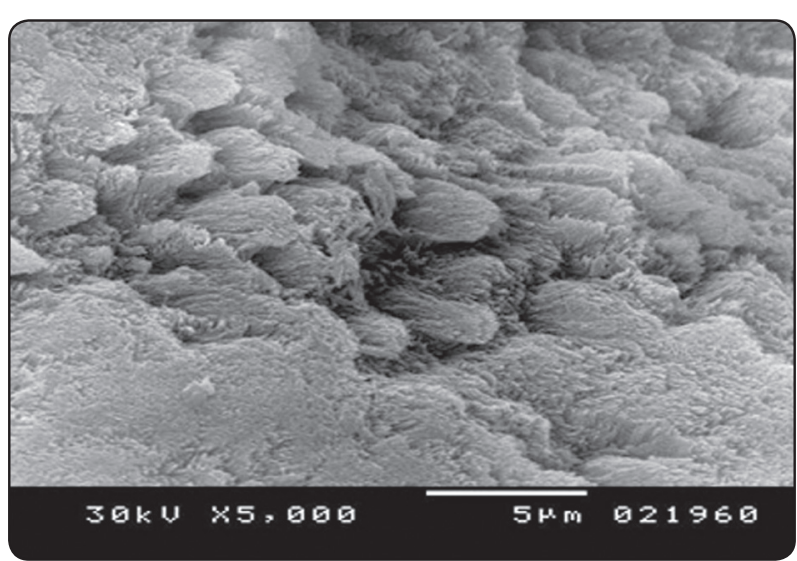

Fig. (1 A) SEM image, 5000x, and showing demineralization of artificially induced white spot demonstrating partial loss of surface minerals.

\section{Statistical analysis}

Levene's test of equality of error variances was performed to test the null hypothesis that error variance in MTBS was similar in tested groups. One way analysis of variance (ANOVA) was selected to analyze the data with 1 within-group factor (method of application of nHA). Bonferroni post hoc test was selected for pair-wise comparisons $(a=.05, n=40)$. Data were analyzed using computer software (SPSS 14.0; SPSS, Inc, Chicago, Ill).

\section{RESULTS}

Cross sectional hardness values showed significant differences between demineralized enamel lesions and sound enamel $(\mathrm{F}=35, \mathrm{P}<.008)$. Mean lesion depth was $377.1 \pm 47.5 \mu \mathrm{m}$ after which hardness values reached a constant plateau equivalent to sound enamel, Figure 1. Mean hardness value of demineralized enamel was $202.6 \mathrm{HVN}$ compared to $380.3 \mathrm{HVN}$ of sound enamel. Scanning electron microscopy revealed characteristic inter-rod dissolution and exposure of enamel rod peripheries resulting in increasing apparent surface roughness, Figure 1-A. After application of the infiltrants, all enamel porosities were sealed, Figure 1-B.

EDX demonstrated that demineralized enamel had a significant reduction of calcium and phos-

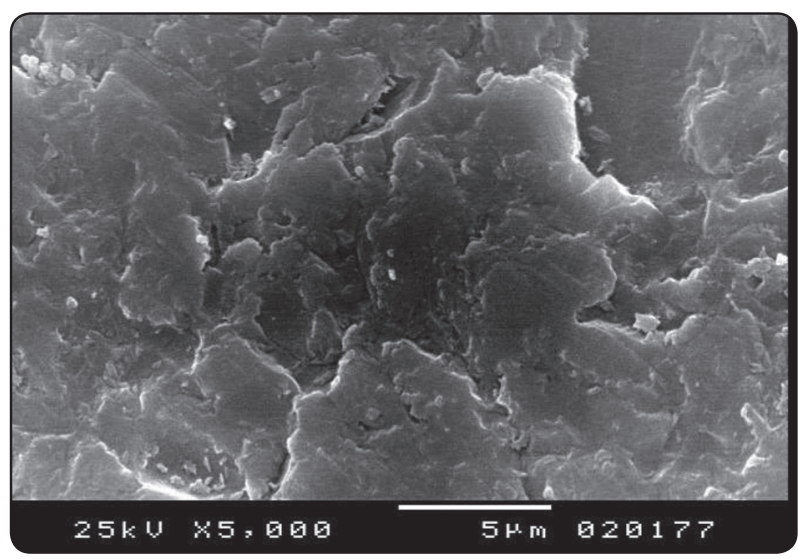

Fig. (1B) SEM image, 5000x, and showing all enamel porosities sealed after application of the infiltrants 
phorous content compared to sound enamel ( $\mathrm{F}=33$, $\mathrm{P}<.005)$. There was a marked reduction of $54 \% \mathrm{wt}$ of both calcium and phosphorous content within the observed lesion. Calcium and phosphorous content reached normal values beyond the observable mean lesion depth. 3 months of water storage resulted in $42 \%$ recovery of calcium content for the directly added nano-particles. This was associated with increase of enamel microhardness to $255 \mathrm{VHN}$. Silanated particles failed to produce similar results.

There were significant statistical differences ( $\mathrm{F}=32, \mathrm{P}<0.001)$ in MTBS values between the tested groups. Direct addition of hydroxyapatite particles reduced MTBS values (19 $\mathrm{MPa} \pm 3.8)$ compared to non-modified adhesive (26 MPa \pm 4.1$)$. On the contrary application of silanated nano particles did not influence MTBS values (24 MPa \pm 3.2 ) compared to the unmodified adhesive. SEM of fractured microbars obtained from different groups showed that direct addition of nHA resulted in agglomeration of the particles on the on the surface of demineralized enamel, Figure 2-A. On the other hand, using silanated particles did not result in creation of structural defects on the surface of demineralized enamel and remained properly distributed in the resin adhesive, Figure 2-B and C.

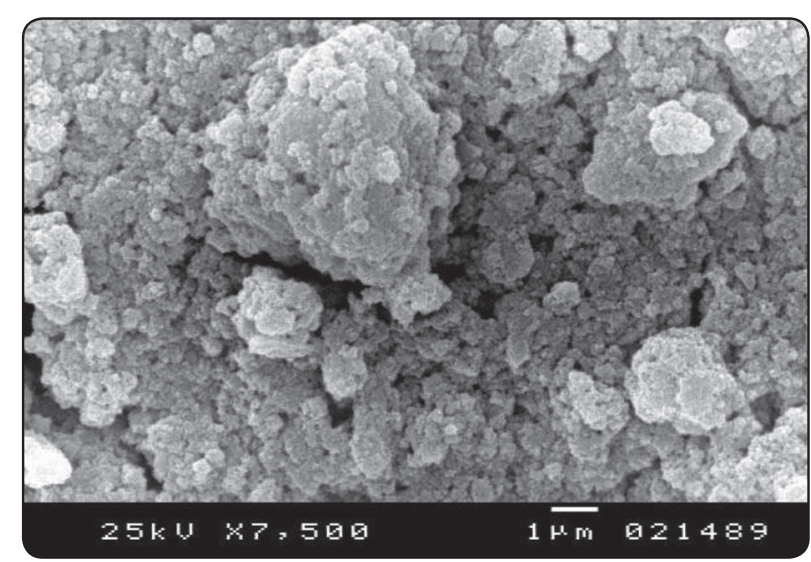

Fig. (2-A) SEM image, 10000x, demonstrating surface appearance of enamel after application of infiltrant.

\section{DISCUSSION}

The selected demineralization model used in this study produced surface features similar to those observed in early enamel carious lesions as the characteristic selective demineralization pattern observed in white spots observed clinically, Figure 1-A, where the inter-rod enamel region was the most affected ${ }^{25}$.

Cross section micro-hardness test revealed a mean lesion depth higher than that reported in literature ${ }^{25,26}$. This may be attributed to the lower $\mathrm{pH}$ used in this study. Demineralized enamel lesions lost almost $46.9 \%$ of its hardness compared to sound enamel with mean lesion hardness of $202.6 \mathrm{HV}$, these results coincide with results reported in literature 27,28 .

Regarding bond strength, direct addition of unsilanized nHA to low viscosity enamel infiltrant resulted in reductions in MTBS values and in clustering of the added particles on top of demineralized enamel. Although nHA could be directly adsorbed on demineralized enamel ${ }^{29,30}$. It seems that bond strength was negatively affected due to absence of good contact between resin adhesive and the added particles. Failure pattern in this group tended to be adhesive between resin adhesive and the clustered particles. On the contrary, unsilanized particles

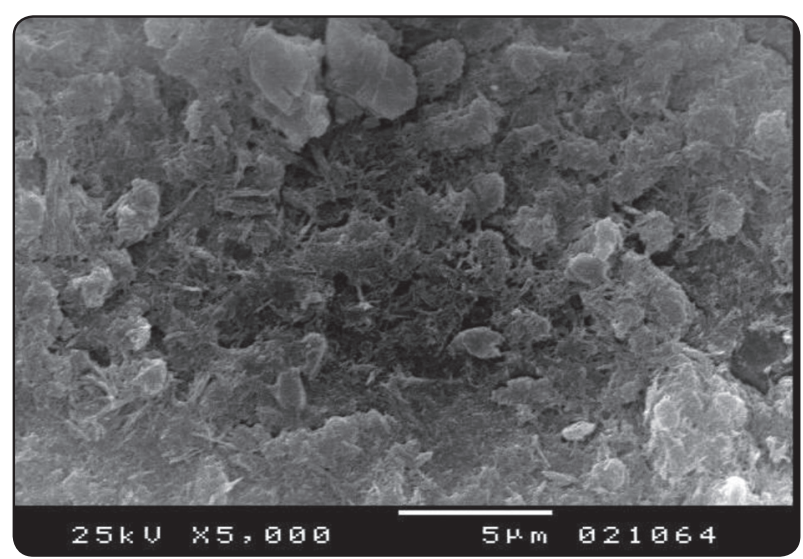

Fig. (2-B) SEM image, 5000x, showing dispersion of unsilanized hydroxy apatite enriched infiltrant 
resulted in increase in calcium uptake causing improved surface hardness of ECE while silanized particles failed to produce similar effect. Silane coating could act as a barrier prevent effective ion exchange with ECE. Further studies are needed to elaborate on this observation.

The incorporation of silanized particles into resin adhesive did not result in deterioration of bond strength to enamel which is in agreement with other studies ${ }^{29,31-34}$. Unfortunately, no results were reported about the effect of mixing nHA with resin adhesives on bond strength to ECE. In the present study $10 \%$ wt nHA was the highest concentration that did not negatively affect bond strength and maintained good contact with demineralized enamel. Several investigations are needed to study the effect of nano hydroxyapatite on remineralization of early enamel lesions and to study the mechanism of ion transfer namely calcium and phosphorous between the particles and the carious enamel surface. TTEMA/ TEGDMA is a low viscosity low shrinkage resin designed to reduce polymerization prestresses ${ }^{16}$. As an enamel infiltrant, these properties may enhance penetration depth to ECE which deserves further investigations ${ }^{35,36}$. With improved remineralization capacity, this resin may offer a simple clinical procedure to treat ECE without the need of further surface treatments.

\section{CONCLUSION}

Within the limitations of this study, addition of $10 \%$ wt silanized nano hydroxyapatite to low shrinkage low viscosity resin did not compromise bond strength to demineralized enamel.

\section{REFERENCES:}

1. Ensanya Ali Abou Neel, Anas Aljabo, Adam Strange, Salwa Ibrahim, Melanie Coathup, Anne M Young, Laurent Bozec, and Vivek Mudera . Demineralization-remineralization dynamics in teeth and bone. Int $\mathrm{J}$ Nanomedicine. 2016; 11: 4743-4763.
2. Vidya Manoharan, R. Krishna Kumar, Arun Kumar Sivanraj, and Selva Balaji Arumugam . Comparative Evaluation of Remineralization Potential of Casein PhosphopeptideAmorphous Calcium Fluoride Phosphate and Novamin on Artificially Demineralized Human Enamel: An In vitro Study. Contemp Clin Dent. 2018 Jun; 9(Suppl 1): S58S63.

3. Radhey Shyam, Manjunath BC, Adarsh kumar, Ridhi Narang, Mamta Ghanghas and Geeta Rani. Role of casein phosphopeptide-amorphous calcium phosphate (cpp-acp) in prevention of dental caries: a review. International Journal of Advanced Research July 2017;5(7):999-1004.

4. Tarang Chadha Arora, Deval Arora, Abhay Mani Tripathi, Gunjan Yadav, Sonali Saha, Kavita Dhinsa. An In-Vitro evaluation of resin infiltration system and conventional pit and fissure sealant on enamel properties in white spot lesions. Journal of Indian Society of Pedodontics and Preventive Dentistry June 2019; 37(2): 133-139.

5. de Sousa FB, Lelis IMP, Figueiredo R, Pires AC, Gerlach RF. Quantitative study of the proportion of the pore volume of human fluorotic enamel filled by resin infiltrant. Arch Oral Biol. 2017;82:134-40.

6. Harleen Kumar, Joseph Emilio Aurelio Palamara , Michael Francis Burrow \& David John Manton . An investigation into the effect of a resin infiltrant on the micromechanical properties of hypomineralised enamel. International Journal of Paediatric Dentistry 2017; 27: 399-411.

7. Eckstein A, Helms HJ, Knosel M. Camouflage effects following resin infiltration of postorthodontic whitespot lesions in vivo: One-year follow-up. Angle Orthod. 2015;85:374-80.

8. Andrej M Kielbassa, Ina Ulrich, Rita Schmidl, Christoph Schüller, Wilhelm Frank, Vanessa D Werth. Resin infiltration of deproteinised natural occlusal subsurface lesions improves initial quality of fissure sealing. Int J Oral Sci. 2017 Jun; 9(2): 117-124.

9. Askar H, Lausch J, Dorfer CE, Meyer-Lueckel H, Paris S. Penetration of micro-filled infiltrant resins into artificial caries lesions. J Dent. 2015;43:832-8.

10. Niwut Juntavee, Apa Juntavee, and Preeyarat Plongniras. Remineralization potential of nano-hydroxyapatite on enamel and cementum surrounding margin of computeraided design and computer-aided manufacturing ceramic restoration. Int J Nanomedicine. 2018; 13: 2755-2765.

11. Aziznezhad M, Alaghemand H, Shahande Z, Pasdar N, Bi- 
jani A, Eslami A, et al. Comparison of the effect of resin infiltrant, fluoride varnish, and nano-hydroxy apatite paste on surface hardness and streptococcus mutans adhesion to artificial enamel lesions. Electron Physician. 2017;9:393442.

12. Andrade Neto DM, Carvalho EV, Rodrigues EA, Feitosa VP, Sauro S, Mele G, et al. Novel hydroxyapatite nanorods improve anti-caries efficacy of enamel infiltrants. Dent Mater. 2016;32:784-93.

13. Bajaj M, Poornima P, Praveen S, Nagaveni NB, Roopa $\mathrm{KB}$, Neena IE, et al. Comparison of CPP-ACP, Tri-Calcium Phosphate and Hydroxyapatite on Remineralization of Artificial Caries Like Lesions on Primary Enamel -An in vitro Study. J Clin Pediatr Dent. 2016;40:404-9.

14. López E, Dominguez J , Gomes G, Mora C, Bittencourt B,Gomes J ,Gomes O.Effect of Conditioning Protocols and Ultrasonic Application of an Infiltrant Resin in White Spot Lesions. Braz. Dent. J. 2019,30(1).

15. Memarpour M, Shafiei F, Rafiee A, Soltani M, Dashti M. Effect of hydroxyapatite nanoparticles on enamel remineralization and estimation of fissure sealant bond strength to remineralized tooth surfaces: an in vitro study.BMC Oral Health. 2019; 19: 92. Published online 2019 May 28. doi: 10.1186/s12903-019-0785-6.

16. Farias de Lacerda AJ, Ferreira Zanatta R, Crispim B, Borges AB, Gomes Torres CR, Tay FR, et al. Influence of de/remineralization of enamel on the tensile bond strength of etch-and-rinse and self-etching adhesives. Am J Dent. 2016;29:289-93.

17. Mohamed A, Wong K, Lee W, Nor M, Hussaini H, Rosli T.In vitro study of white spot lesion: Maxilla and mandibular teeth. Saudi Dent J. 2018 Apr; 30(2): 142-150.

18. Gelani R, Zandona A, Lippert F, Kamocka M, Eckert G. In Vitro Progression of Artificial White Spot Lesions Sealed With an Infiltrant Resin. Oper Dent. 2014 SepOct;39(5):481-8.

19. Yim HK, Min JH, Kwon HK, Kim BI. Modification of surface pretreatment of white spot lesions to improve the safety and efficacy of resin infiltration. Korean J Orthod. 2014 Jul; 44(4): 195-202.

20. Zhou Y, Matin K, Shimada Y, Sumi Y, Tagami J. Evaluation of resin infiltration on demineralized root surface: An in vitro study. Dent Mater J. 2017;36:195-204.

21. Colak H, Ercan E, Hamidi M.Shear bond strength of bulk- fill and nano-restorative materials to dentin. Eur J Dent. 2016 Jan-Mar; 10(1): 40-45.

22. ten Cate JM. Models and role models.Caries Res. 2015;49 Suppl 1:3-10.

23. Ferro AC, Guedes M. Mechanochemical synthesis of hydroxyapatite using cuttlefish bone and chicken eggshell as calcium precursors. Mater Sci Eng C Mater Biol Appl. 2019;97:124-140.

24. Yang H, Chen Z, Yan H, Huang C.Effects of calcium-containing desensitizers on the bonding stability of an etchand-rinse adhesive against long-term water storage and $\mathrm{pH}$ cycling. Dent Mater J. 2018 Jan 30;37(1):122-129.

25. Vongsavan K, Rirattanapong P, Surarit R. Comparison of Children's Follow-on Instant Powdered Cow's Milk Formula, Buffalo Milk Formula and Chicken-Based Formula on Enamel Microhardness of Bovine Teeth in Vitro. Southeast Asian J Trop Med Public Health. 2016;47:328-33.

26. Mohammadi N, Farahmand Far MH. Effect of fluoridated varnish and silver diamine fluoride on enamel demineralization resistance in primary dentition. J Indian Soc Pedod Prev Dent. 2018;36:257-61.

27. Lopes C, Soares C, Lara V, Arana-Chavez V, Soares P, Novais V.Effect of fluoride application during radiotherapy on enamel demineralization .J Appl Oral Sci. 2019; 27: e20180044.

28. Eskelsen E, Catelan A, Hernades N, Soares L, Cavalcanti A, Aguiar F, Liporoni P.Physicochemical changes in enamel submitted to $\mathrm{pH}$ cycling and bleaching treatment. Clin Cosmet Investig Dent. 2018; 10: 281-286.

29. Costa AR, Correr-Sobrinho L, Ambrosano GM, Sinhoreti MA, Borges GA, Platt JA, et al. Dentin bond strength of a fluoride-releasing adhesive system submitted to $\mathrm{pH}$-cycling. Braz Dent J. 2014;25:472-8.

30. Rabelo NJ, Jacqueline S, Monica Y, Sartori MJ, LimaSS. Pretreatment with Sodium Fluoride Maintains Dentin Bond Strength of a Two-Step Self-Etch Adhesive after Thermal Stressing.J Adh Dent. 2017,19(6):517-523.

31. Abuna G, Feitosa VP, Correr AB, Cama G, Giannini M, Sinhoreti MA, et al. Bonding performance of experimental bioactive/biomimetic self-etch adhesives doped with calcium-phosphate fillers and biomimetic analogs of phosphoproteins. J Dent. 2016;52:79-86.

32. Barbosa-Martins LF, Sousa JP, Alves LA, Davies RPW, Puppin-Rontanti RM. Biomimetic Mineralizing Agents 
Recover the Micro Tensile Bond Strength of Demineralized Dentin. Materials (Basel). 2018;11.

33. Kim J, Vaughn RM, Gu L, Rockman RA, Arola DD, Schafer TE, et al. Imperfect hybrid layers created by an aggressive one-step self-etch adhesive in primary dentin are amendable to biomimetic remineralization in vitro. $\mathrm{J}$ Biomed Mater Res A. 2010;93:1225-34.

34. Zhang Y, Wang Z, Jiang T, Wang Y. Biomimetic regulation of dentine remineralization by amino acid in vitro. Dent
Mater. 2019;35:298-309.

35. Altmann ASP, Collares FM, Balbinot GS, Leitune VCB, Takimi AS, Samuel SMW. Niobium pentoxide phosphate invert glass as a mineralizing agent in an experimental orthodontic adhesive. Angle Orthod. 2017;87:759-65.

36. Yue S, Wu J, Zhang Q, Zhang K, Weir MD, Imazato S, et al. Novel dental adhesive resin with crack self-healing, antimicrobial and remineralization properties. J Dent. 2018;75:48-57. 